\title{
Eximerlaser mit gauß-förmigem Strahlprofil
}

\author{
Prof. Dr. Harald Gündel †, Hans Volkmann
}

\subsection{Ziel der Projektarbeiten}

Wegen ihrer Wellenlänge im ultravioletten Spektralgebiet gehören Eximerlaser (z. B. KrF, ArF, XeCl) zu den am vielseitigsten eingesetzten Lasern in der Forschung und zunehmend auch in der industriellen Anwendung. Alle am Markt erhältlichen Excimer-Laser weisen jedoch einen wesentlichen Nachteil auf, der ihre Einsatzmöglichkeiten begrenzt.

Bedingt durch konstruktive Besonderheiten ist ihre Strahlqualität unbefriedigend. Sie weisen einen rechteckförmigen Strahlquerschnitt auf mit einem komplizierten Gemisch verschiedener transversaler Moden (Multimodenstruktur). Diese Eigenschaft wirkt sich negativ auf die Fokussierbarkeit der Strahlung aus, wodurch der positive Einfluß der kurzen Wellenlängen z. T. wieder zunichte gemacht wird. Besonders störend ist dieser Effekt z. B. beim Bohren feinster Löcher oder beim Einkoppeln der Strahlung in Lichtleiter.

Das Ziel der Arbeiten besteht daher darin, einen Eximerlaser zu entwickeln, der die Vorteile der kurzen Wellenlänge mit einer guten Strahlqualität verbindet. Anzustreben ist ein kreisrunder Strahlquerschnitt mit nur einer Strahlungsmode und Gauß-Profil. Dabei sollen die Erfahrungen genutzt werden, die im Institut für Plasmaund Lasertechnik der TFH Wildau bei der Entwicklung von Stickstofflasern mit gauß-ähnlichem Strahlprofil gewonnen wurden. Ein solches Gerät würde die Einsatzgebiete von Eximer-Lasern erweitern und hätte voraussichtlich gute Marktchancen.

\subsection{Beschreibung der ausgeführten Arbeiten}

\subsubsection{Lösungsweg}

Die Strahlqualität (Modenstruktur) eines jeden Gaslasers wird von der Geometrie des aktiven Mediums und den Eigenschaften des verwendeten Resonators bestimmt. In allen zur Zeit auf dem Markt erhältlichen Eximerlasern wird dieses aktive Medium durch eine schnelle Impulsentladung gebildet, die zwischen zwei einander gegenüberstehenden nahezu plattenförmigen Elektroden brennt. Damit hat das aktive Medium einen für eine gute Strahlqualität (kreisrunde Geometrie-TEM ${ }_{00}$ Mode) ungünstigen rechteckförmigen Querschnitt, bei dem die Breite ein Vielfaches der Höhe beträgt. Diese Form wurde bisher durch die geforderten kurzen Stromimpulse und die daher notwendigen niedrigen Induktivitäten erzwungen. Zur Erzeugung kreisrunder Laserstrahlung sind prinzipiell zwei Wege möglich:
- Nutzung gefalteter Resonatoren, bei den das gesamte aktive Medium „nacheinander" durch einen gaußähnlischen Strahl abgerufen wird.

- Entwicklung einer Entladungsanordnung, die von vornherein ein aktives Medium mit nahezu kreisrunden Querschnitt erzeugt.

In den nachfolgend beschriebenen Arbeiten wurde der zweite Weg beschritten. Dafür war ausschlaggebend, daß bereits Erfahrungen bei der Herstellung der geforderten zylindersymmetrischen Entladungsgeometrie im Fall von Stickstofflasern vorlagen. Derartige Laser mit nahezu kreisförmigen Strahlquerschnitt wurden bereits in kleinen Stiickzahlen gefertigt und auf den Markt gebracht. Dabei wird die Tatsache ausgenutzt, daß Entladungen, die zwischen zwei stiftförmigen Elektroden brennen, von vornherein eine Zylindersymmetrie aufweisen. Wegen der geforderten geringen Induktivitäten ist dabei die Länge der Entladung (Elektrodenabstand) auf wenige Zentimeter begrenzt, also zu klein, um eine ausreichende optische Verstärkung zu erreichen. Die Lösung besteht daher darin, elektrisch parallele Längsentladungen zwischen zwei Stiftelektroden optisch hintereinander anzuordnen. (vgl. Abb. 1)

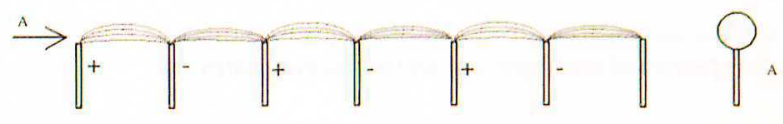

Abb.1: Multieelektroden-Längs-Anordnung

Dabei wechseln die Elektroden in ihrer Polarität. Jede Elektrode versorgt zwei benachbarte Elektrodenkanäle. Eine einfache Nutzung dieser für Stickstofflaser eingesetzten Anordnung für Eximerlaser ist allerdings nicht möglich.

In $\mathrm{N}_{2}$-Laser werden Drücke von einigen 10 mbar verwendet, während Eximerlaser in Gasgemischen von mehreren bar betrieben werden muissen. Das macht u. a. den Einsatz einer Vorionisation erforderlich. Außerdem verlangen die aggressiven halogenhaltigen Eximer-Lasergase eine spezielle Gefäßtechnologie und ein spezielles Gashandling.

Aus diesem Grund wurde bei der Entwicklung auf die in bisher produzierten Eximerlaser bewährte Gefäßtechnologie zuruick gegriffen. Ziel war es, ein vorhandenes Lasergefäß mit einem neu zuentwickelnden Elektrodensystem auszurüsten und zu optimieren. Dazu mußte die gesamte Stromversorgung und die notwendige Steuerelektronik neu entwickelt und aufgebaut werden. Schließlich wurde die Gasversorgung für die Durchfuihrung der Experimente neu erstellt. 


\subsubsection{Aufbau einer Anlage zur Gasversorgung}

Mit einer Vakuumpumpe kann sowohl die Mischanlage als auch das Lasergefäß evakuiert werden. Ein Filter am Gasaustritt der Pumpe hält die giftigen Halogenide zuriick und das gereinigte Gas wird in die Abluftanlage des Labors gefuihrt. In der Gasmischanlage können bis zu fünf verschiedene Gase miteinander kombiniert werden. Über Reduzier- und Dosierventile werden die einzelnen Gaskomponenten mit unterschiedlichen Druicken in das Lasergefäß eingelassen, so daß am Ende das geforderte Gasgemisch zur Verfügung steht. Die gesamte Anlage wurde unter Nutzung von Swage-Log-Verbindungsteilen in Edelstahltechnik aufgebaut und mit einem Helium-Lecksuchgerät auf Dichtheit überprüft.

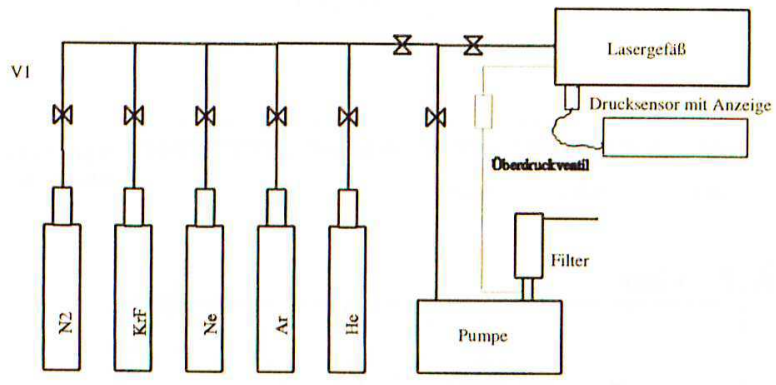

Abb. 2: Gasmischanlage

\subsubsection{Konstruktion und Bau des Elektrodensystems}

Hierzu waren zwei Hauptprobleme zu lösen:

Integration eines Systems zur Vorionisation in die Multielektroden-Längsanordnung

Dazu wurden anstelle der Stiftelektroden V-förmig angeordnete Elektrodenpaare verwendet, Zwischen denen die Vorionisationsfunken gezündet werden sollen.

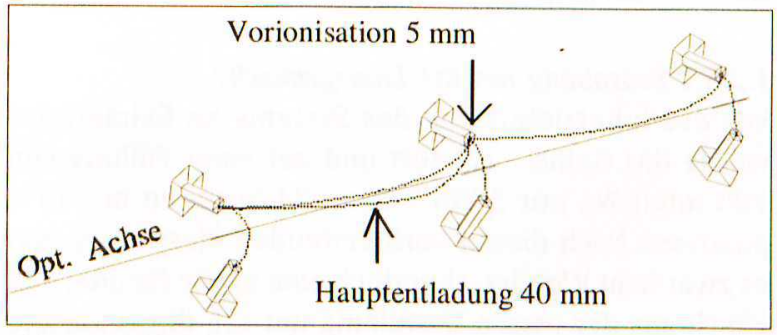

Abb. 3: Stiftelektrodenanordnung mit Vorionisationselektroden

Die Elektrodenpaare wurden im Abstand von $40 \mathrm{~mm}$ längs der optischen Achse angeordnet, so daß jeweils eine der Elektroden die Laserentladung versorgt. Bei 11 Elektrodenpaaren ergibt sich damit eine Länge des aktiven Mediums von $400 \mathrm{~mm}$

\section{Entwicklung vakuumdichter und korrosionsbeständiger Elektrodendurchfïhrungen}

Die Elektroden werden durch den vorhandenen PVDFDeckel des Lasergfäßes gefuihrt. Sie bestehen aus $2 \mathrm{~mm}$ dicken und $100 \mathrm{~mm}$ langen Ni-Draht. Die Abdichtung erfolgt mit Hilfe eines Viton-Nullrings, der mittels einer durchbohrten Schraube in die PVDF-Platte gepreßt wird. Diese Konstruktion gewährleistet eine lösbare Verbin- dung. Sollte nach mehrmaligem Wechseln der Elektroden das Gewinde im PVDF-Block nicht mehr in Ordnung sein, kann ein Gewindeeinsatz M 8 aus Messing eingeschraubt werden.

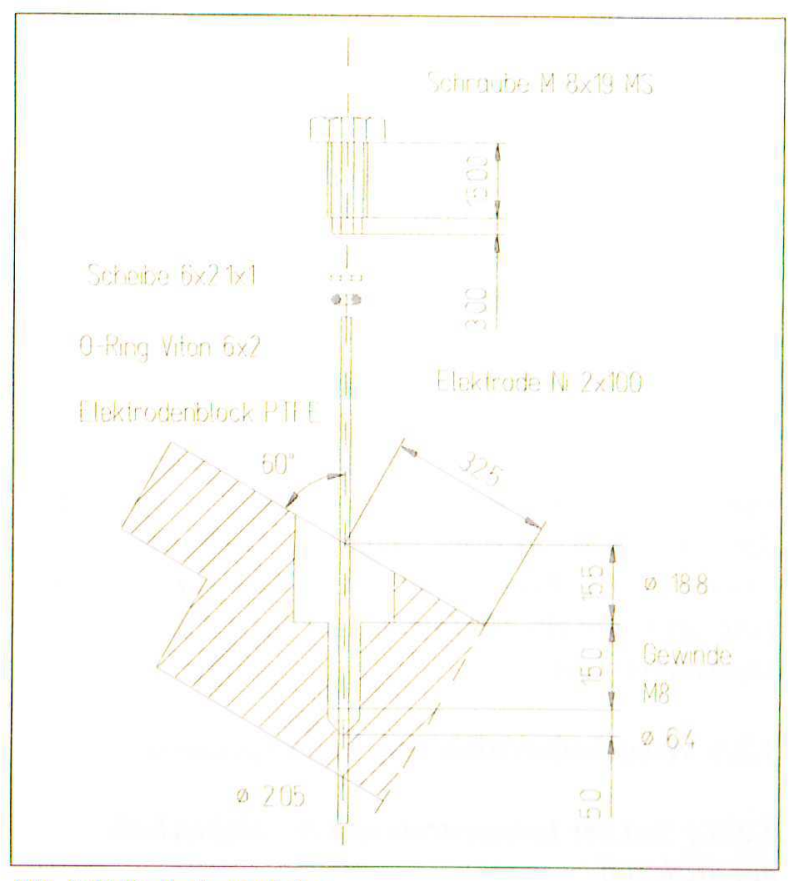

Abb. 4: Elektrodenkonstruktion

\subsubsection{Entwicklung der Stromversorgung und Steuerung des Lasers}

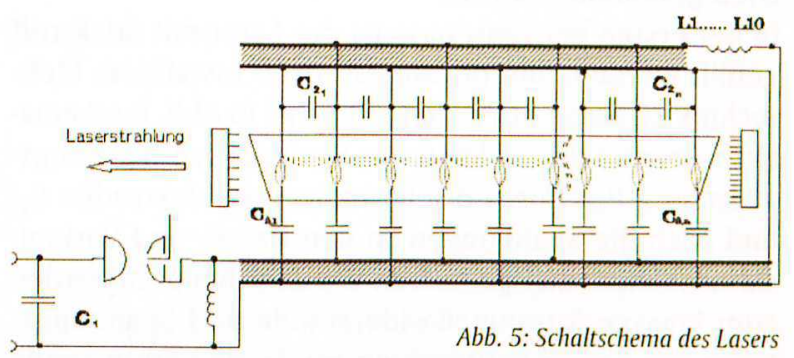

Die Abbildung 5 zeigt den prinzipiellen Aufbau der elektrischen Anordnung der Bauelemente. Der Speicherkondensator C1 wird von einer Spannungsquelle aufgeladen. Wird der Hochspannungsschalter (eine Dreielektroden-Funkenstrecke oder ein Thyratron) geschlossen, entlädt sich der Kondensator C1 in die Kondensatoren $\mathrm{C}_{1-10}$ und C $3_{1-10}$. Die Spannung an den Elektroden des Lasergefäßes steigt bis zur Durchschlagsspannung an, die von dem Gas, dem Gasdruck, der Elektrodenform und der Spannungsanstiegsgeschwindigkeit abhängt. Wird der Kondensator C1 sehr schnell uiber den Schalter in die Kondensatoren $\mathrm{C}_{\mathrm{n}}$ und $\mathrm{C} 3_{\mathrm{N}}$ umgeladen, kommt es zu einer Spannungsüberhöhung an den Elektroden, die weitaus höher als die statische Durchschlagsspannung liegt. Es wird so mehr Energie in das Entladungsplasma eingespeist. Die schnelle Umladung des Kondensators C1 in die Kondensatoren C2 und C3 erfolgt über einen Bandleiter. Er besteht aus einer Acrylglasplatte (5 mm stark) als Dielektrikum und zwei 150 mm breiten Cu-Platten. An einer Längsseite der Platten sind die Abgriffe zum Aufladen der Kondensatoren $\mathrm{C}_{\mathrm{n}}$ und $\mathrm{C}_{\mathrm{n}}$ angebracht. 
Der Laser wird zunächst mit einer Drei-ElektrodenFunkenstrecke an einem $40 \mathrm{kV}$ Netzgerät von ALE betrieben. Erst später, nach der Optimierung der einzelnen Komponenten des Entladungskreises und Anpassung des Entladungsnetzwerks an den Plasmawiderstand, kann ein Thyratron als Hochspannungsschalter eingesetzt werden.

Von einem kleinen Pult aus wird der Laser gesteuert. Die Betriebsarten („kontinuierlicher Betrieb“, „,kontinuierlicher Betrieb uiber Taste“ und „externe Triggerung“) sowie die Folgefrequenz können am Pult gewählt und der Laser mit zwei Tasten ein- und ausgeschaltet werden. Die Steuerelektronik im Geräteeinschub schaltet vor jedem Laserimpuls die Versorgungsspannung für das Lasersystem ein. Sobald die eingestellte Betriebsspannung von maximal $40 \mathrm{kV}$ erreicht ist, wird ein Laserimpuls ausgelöst und die Hochspannung abgeschaltet. In der Betriebsart „externe Triggerung“ befindet sich das Lasersystem in Bereitschaft, die Hochspannung ist eingeschaltet und ein externes Triggersignal löst sofort den Laserimpuls aus.

\subsubsection{Versuchsdurchführung und Ergebnisse}

\subsubsection{Test des Lasersystems und der Meßtechnik mit Stickstoff}

Die gesamte Anlage wurde mit einem Helium-Lecksuchgerät sowohl unter Vakuum als auch bei Überdruck (5 bar) auf Dichtheit gepriift. Es konnten keine undichten Stellen festgestellt werden, so daß der Laser in Betrieb genommen wurde.

In der ersten Betriebsphase ist der Laser mit Stickstoff gefuillt worden, um die am Laserrohr installierte Meßtechnik zu testen. Der Meßaufbau ist in Abb. 6 schematisch dargestellt. Es kann der Strom durch die Hauptentladung $\left(i_{\mathrm{e}}\right)$, durch die Vorionisationselektroden $\left(i_{v}\right)$ und auch die Spannungen an den Haupt- und Vorionisationselektroden gemessen werden. Benutzt werden zum Messen Strommeßwiderstände und Spannungsteiler aus eigner Entwicklung sowie eine Stromsonde von Pearson (Typ 2878). Der Laserimpuls wird über verschiedene Strahlteiler geschwächt auf eine Vacuumphotodiode (ATL, Typ A-5005-C) geschickt. Die Signale der Strommeßwidwerstände, des Spannungsteilers und der Photodiode werden zeitaufgelöst auf einen Oszillografen (Tektronix TDS 620) dargestellt. Die Energie eines Laserimpulses wird mit einem Pyroelektrischen Energiemeßgerät (RDA, PEM 521) gemessen. Im Stickstofflaserbetrieb wurde der Rückkoppelspiegel und das Austrittsfenster des Lasergefäßes sowie ein externer Spiegel (Aluminium) justiert.

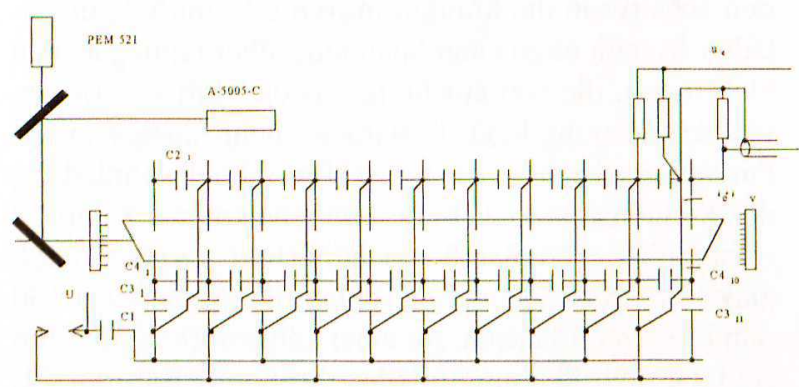

Abb. 6: Meßaufbau
In der Abbildung 7 sind fuir den Stickstofflaser typische Oszillogramme dargestellt. Es zeigte sich, daß die eingesetzte Meßtechnik zur Zufriedenheit arbeitet und Resultate liefert, die mit fruiheren Messungen an longitudinalangeregten Stickstofflasern übereinstimmen.
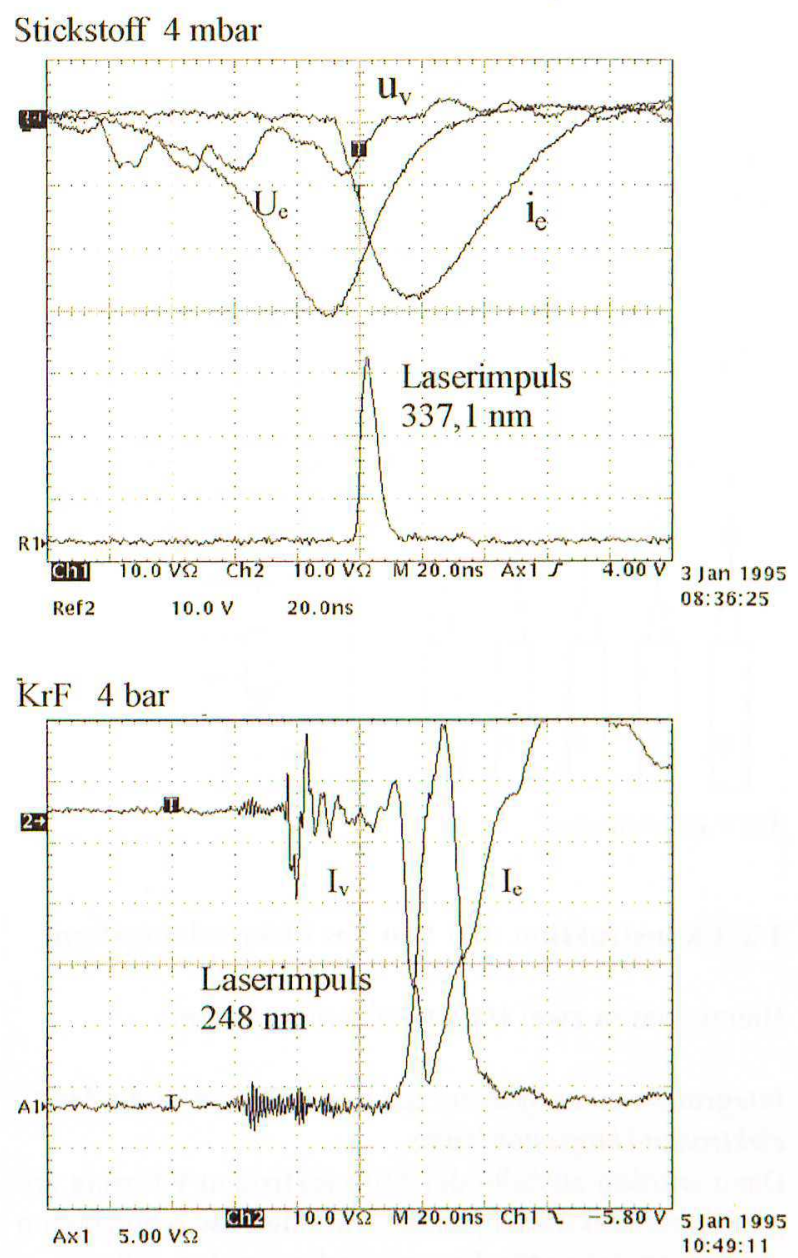

Abb. $7: \mathrm{N}_{2}$-Laser (oben) und KrF-Laser (unten)

\subsubsection{Erprobung des KrF-Lasergemischs}

Vor des Inbetriebnahme des Systems als Eximerlaser wurde das Gefäß evakuiert und mit einer Füllung von 100 mbar Ne mit $5 \mathrm{Vol} \% \mathrm{~F}_{2}$ in 24 Stunden bei $45^{\circ} \mathrm{C}$ passiviert.Nach diesen vorbereitenden Messungen war es zwar kein Wunder, aber doch eine große Freude, daß gleich aus der ersten Gasfüllung mit KrF die erwartete Laserstrahlung bei $248 \mathrm{~nm}$ erfolgte. Die Abbildung 8 zeigt eine der ersten Messungen des Entladungsstroms und des Laserimpulses bei einer Wellenlänge von 248 nm. Deutlich ist der Einsatz der Vorionisation ca. $30 \mathrm{~ns}$ vor dem Beginn des Stroms durch die Hauptentladung zu erkennen.

In zahlreichen Versuchsreihen wurden die äußeren Kreisparameter variiert und ihr Einfluß auf die Parameter des Laserimpulses (Ausgangsenergie, Halbwertsbreite und Stabilität der Entladung) untersucht. Desweiteren wurde die Gaszusammensetzung und der Gasdruck verändert. Die besten Ergebnisse wurden bei einer Ladespannung von $40 \mathrm{kV}, \mathrm{C} 1=10 \mathrm{nF}, \mathrm{C} 2_{1-10}=1 \mathrm{nF}$, $\mathrm{C}_{1-11}=150 \mathrm{pF}$ und einer Verzögerung der Hauptentladung gegenüber der Vorionisation von ca. 50 ns erreicht. Die Impulsenergie betrug etwa $12 \mathrm{mWs}$ bei ei- 
ner Halbwertsbreite von 14 ns. Damit konnte ein Eximerlaser mit runder Strahlgeometrie realisiert werden. Der eingeschlagene Entwicklungsweg hat sich als gangbar und vielversprechend erwiesen.

\subsection{Bewertung der erzielten Ergebnisse}

Es wurde gezeigt, das unter Nutzung vorhandener Baugruppen, Adaptierung einer neuen Elektrodenanordnung und konsequenter Anwendung der Kondensatorumladung über ein Cu-Bandleitersystem, ein Eximerlaser mit annähernd gaußförmigen Strahlprofil und akzeptabler Ausgangsenergie von etwa $12 \mathrm{~mJ}$ bei einer aktiven Länge von $400 \mathrm{~mm}$ aufgebaut werden kann.

Damit wurde das hauptsächliche Ziel der Entwicklungsarbeiten erreicht und die Voraussetzungen für weitergehende Untersuchungen mit dem Ziel einer Fertigungsaufnahme geschaffen.

Zu diesen Untersuchungen gehören insbesondere

- Gewährleistung der Reproduzierbarkeit und Stabilität der Laserstrahlung,

- Erhöhung der Folgefrequenz.

Beide Probleme können durch Optimierung der Vorionisation und den Einbau eines konventionellen Gasumwälzsystems gelöst werden.

\section{Beseitigung der elektromagnetischen Störstrahlung}

Neben schon realisierten Maßnahmen (Einbau der Steuerelektronik, der Druckanzeige und der Sicherheitsschaltung in einem Geräteeinschub an der Stirnseite des Lasergehäuses, Abblockung der Hochspannungszufuihrung mit LC-Filter) kann das durch Einbau der Hochspannungsversorgung in das Gehäuse realisiert werden. Eine weitere Verbesserung der Standzeit des Lasergemisches ist durch Einsatz einer neuartigen Variante der Vorionisation (stille Entladung) denkbar, die erst in neuester Zeit bekannt geworden ist.

Die durchgeführten Untersuchungen wurden von der Firma Radiant Dyes Laser \& Accessories GmbH und dem Land Nordrhein-Westfalen unterstiitzt.

\section{Verfasser}

Prof. Dr. Harald Gündel †

Hans Volkmann

Technische Fachhochschule Wildau

Fachbereich Physikalische Technik

Tel. (0 33 75) 507-121 